\title{
TWENTY-SIXTH ANNUAL SASKATCHEWAN CHRISTMAS BIRD COUNT, 1967
}

\section{Compiled by Mary Houston, 863 University Drive, Saskatoon}

Christmas bird counts were taken in 25 areas in Saskatchewan in 1967, producing interesting results.

A Mockingbird, rare in the province at any time, was reported from Regina. At Val Marie, a Fox Sparrow was seen-not on count day, but during the count period. These two species will be added to the all-time list for Christmas bird counts in Saskatchewan and bring the total to 115 species.

Aside from the waterbirds (White Pelican, Mute and Whistling Swans, Canada Goose, Mallard, Gadwall, Pintail, Shoveler, Canvasback and Common Goldeneye) a large number of species we consider "summer birds" were counted. These included, as well as the Mockingbird and Fox Sparrow, such species as Yellow-shafted Flicker (seen in 5 areas), Robin (2 areas), Cedar Waxwing (2 areas) and Redwinged Blackbird, Common Grackle, Rusty Blackbird and Tree Sparrow (1 area each).

A total of 57 species was seen on count days with an additional five species seen during the count period.

Every one of the 25 areas reporting recorded Black-billed Magpies; 24 recorded House Sparrows; 20, Snow Buntings; and 19, Black-capped Chickadees.

Stan Riome of Nipawin coordinated a survey of the Nipawin area with the $\mathrm{N}$ ipaw in-Squaw Rapids, NipawinWhite Fox and Codette regions being covered by separate groups. The seven Bald Eagles: reported from the Nipawin-Squaw Rapids area were of particular interest.

Skull Creek counters, with Steve Mann as compiler, have a pleasant tradition of finishing off their count with dinner and an evening of comparing notes, at the home of one of the counters.

BROADVIEW, Dec. 26; 75 miles by car and 1 on foot in 6 hours; temp. $-10^{\circ}$; wind NW $12 \mathrm{mph}$; 5 species, 84 individuals. Sharp - tailed grouse, 5; Black-billed Magpie, 53; Black-capped Chickadee, 5; Starling, 15; House Sparrow, 6.-D. G. Francis.

CODETTE, Dec. 31 ; temp. $-30^{\circ}$; 21 species, 224 individuals. Goshawk, 1; Spruce Grouse, 2; Ruffed Grouse, 4; Sharp-tailed Grouse, 2; Gray Partridge, 18; Rock Dove, 4; Great Horned Owl, 1; Snowy Owl, 2; Boreal Owl, 1; Hairy Woodpecker, 1; Downy Woodpecker, 1; Blue Jay, 2; Black-billed Magpie, 6; Common Raven, 4; Blackcapped Chickadee, 6; Bohemian Waxwing, 8; Starling, 12; House Sparrow, 28; Pine Grosbeak, 7; Common Redpoll, 14; Snow Bunting, 100.-W. Harstad.

CRAVEN, Dec. 23; 52 miles by car and 3 miles on foot in $4 \frac{1}{2}$ hours; temp. $-5^{\circ}$ to $5^{\circ}$; wind NW $20 \mathrm{mph}$; mostly overcast with occasional light snow; 2 inches of snow; 10 species, 517 individuals. Rough-legged Hawk, 1; Rock Dove, 12; Short-eared Owl, 1; Horned Lark, 45; Black-billed Magpie, 15; Black-capped Chickadee, 3; Bohemian Waxwing, 304; Starling, 2; House Sparrow, 74; Snow Bunting, 60. (Add: Ring-necked Pheasant, 1, Dec. 28; Northern Shrike, 1, Dec. 30; Evening Grosbeak, 12, Dec. 29).-Greg Bobbitt, Eric Cooke.

DILKE, Dec. 28; 70 miles by car and 2 on foot in $3 \frac{1}{2}$ hours; temp. $10^{\circ}$ to $16^{\circ}$; wind $\mathrm{SW}$ at $25 \mathrm{mph}$ shifting to $\mathrm{NW}$ at $25 \mathrm{mph} ; 6$ inches of snow heavily drifted; 6 species, 289 individuals. Gray Partridge, 1; Horned Lark, 91; Black-billed Magpie, 27; Black-capped Chickadee, 1; Bohemian Waxwing, 144; House Sparrow, 25. (Add: Sharp-tailed Grouse, 1, Dec. 23; Snowy Owl, 2, Dec. 23 and 26).Boswell Belcher (compiler), Margaret Belcher, Mr. and Mrs. S. R. Belcher.

FORT QU'APPELLE, Dec. 27; 13 species, 163 individuals. Sharp-tailed 
Grouse, 6; Gray Partridge, 9; Rock Dove, 12; Downy Woodpecker, 1; Gray Jay, 1; Black-billed Magpie, 3; Blackcapped Chickadee, 4; Robin, 1; Bohemian Waxwing, 6; House Sparrow, 18; Pine Grosbeak, 1; Slate-colored Junco, 1; Snow Bunting, 100. (Add: Ruffed Grouse, 1, Dec. 24 and 28; Great Horned Owl, 1, Dec. 23 and Dec. 26; Hairy Woodpecker, 2, Dec. 21; Blue Jay, 1, Dec. 22 and 4, Dec. 28; Whitebreasted Nuthatch, 1, Dec. 23; Northern Shrike, 1, Jan. 1).-Dr. G. D. Barnett, E. Manley Callin (compiler), Mr. and Mrs. Errol Cochrane, Mr. and Mrs. William Gray, Ron Hooper, Dick Nevard, Horace Reed, Sham Regan, Chad Sunley.

GIRVIN, Dec. 28 ; 6 species, 436 individuals. Golden Eagle, 1; Sharp-tailed Grouse, 7; Black-billed Magpie, 2; Starling, 1; House Sparrow, 250; Snow Bunting, 175. (Add: Gray Partridge, 9, Dec. 31; Snowy Owl, 1, Dec. 22; Horned Lark, 5 on Dec. 21, 25 on Dec. 22, 40 on Dec. 23, 125 on Dec. 25; Northern Shrike, 1, Dec. 22).-Fred Kochendorfer.

GRENFELL, Dec. 31; 6 miles by car and around the yard throughout the day; temp. $-13^{\circ}$; wind SE $15 \mathrm{mph} ; 9$ inches of snow; 4 species, 28 individuals. Sharp-tailed Grouse, 6; Blackbilled Magpie, 2; House iSparrow, 100; Snow Bunting, 20. (Add: Ruffed Grouse, 2, Dec. 24; Great Horned Owl, 1, Jan. 1; Black-capped Chickadee, 1, Jan. 1).-Mr. and Mrs. John Hubbard. HAWARDEN, Dec. 21; 20 miles by car and on foot; temp. $10^{\circ} ; 3$ inches of snow; 3 species, 62 individuals. Horned Lark, 40; Black-billed Magpie, 2; House Sparrow, 20. (Add: Snowy Owl, 1, Dec. 25; Bohemian Waxwing, 40, Dec. 20; Snow Bunting, 1000, Dec. 27).-Harold Kvinge.

KENASTON, Dec. 28; by car, by horse and on foot covering tharoughly 480 acres; temp. $-4^{\circ}$ to $23^{\circ}$; heavily overcast; wind light, rising to $15 \mathrm{mph} ; 8$ inches of snow; 9 species, 94 individuals. Sharp-tailed Grouse, 2; Gray Partridge, 30; Great Horned Owl, 1; Blue Jay, 3; Black-billed Magpie, 14; Black-capped Chickadee, 2; House Sparrow, 40; Slate-colored Junco, 1;
Snow Bunting, 1. (Add: Golden Eagle, 1, Dec. 24; Rock Dove, 40; Horned Lark, 15 on Dec. 22 and 15 on Jan. 1.)-Lawrence Beckie.

LEADER, Dec. 27; 1 hour in the field; temp. $20^{\circ}$; clear and calm; 4 inches of snow; 5 species, 220 individuals. Sharptailed Grouse, 5; Ring-necked Pheasant, 2; Blue Jay, 3; Black - billed Magpie, 10; Bohemian Waxwing, 200. -Daisy D. Meyers.

MOOSE JAW, Dec. 26; 90 miles by car and 10 miles on foot; temp. $-5^{\circ}$ to $-10^{\circ}$; wind $10 \mathrm{mph}$; sunny; 8 inches of snow; 11 species, 748 individuals. Ring-necked Pheasant, 10; Gray Partridge, 11; Rock Dove, 33; Yellowshafted Flicker, 2; Black-billeả Magpie, 27; Black-capped Chickadee, 19; Bohemian Waxwing, 302; Starling, 5; House Sparrow, 134; Pine Grosbeak, 42; Snow Bunting, 163. (Add: Hairy Woodpecker, 2, Dec. 27; Downy Woodpecker, 2, Dec. 27; Northern Shrike, 1, Dec. 21).-Bev. Brander, Mrs. A. Davis, Carl Ellis, Pat Kern, Mr. and Mrs. Cy Knight, Walter Riome, M. Ritchie, Mr. and Mrs. Gordon Silversides, Ruth and Bob Tobaka, Mrs. Waterson, Lee Woodley. (Members of the Moose Jaw Natural History Society. Leith Knight, compiler.)

NIPAWIN-SQUAW RAPIDS, Dec. 26; all day; temp. $-10^{\circ}$; wind NW 10 mph; 23 species, 429 individuals. Common Goldeneye, 21; Goshawk, 1; Golden Eagle, 1; Bald Eagle, 7; Rock Dove, 22; Snowy Owl, 3; Hairy Woodpecker, 4; Downy Woodpecker, 1; Gray Jay, 3; Blue Jay, 4; Black-billed Magpie, 27; Common Raven, 12; Black-capped Chickadee, 9; Boreal Chickadee, 5; Red-breasted Nuthatch, 1; Northern Shrike, 1; Starling, 12; House Sparrow, 60; Evening Grosbeak, 50; Pine Grosbeak, 4; Hoary Redpoll, 1; Common Redpoll, 30; Snow Bunting, 150. - David Riome, Stan Riome.

NIPAWIN - WHITE FOX, Dec. 31; temp. $-30^{\circ} ; 12$ species, 67 individuals. Sharp-tailed Grouse, 12; Gray Partridge, 3; Hairy Woodpecker, 2; Downy Woodpecker, 1; Gray Jay, 3; Blue Jay, 1; Black-billed Magpie, 2; 
Common Raven, 13; Black-capped Chickadee, 3; House Sparrow, 2; Pine Grosbeak, 3; Snow Bunting, 22.-Anne Matthew, Walter Matthews, Wm. Matthews.

NOKOMIS, Dec. 26; 40 miles by car; $2 \frac{1}{2}$ hours; temp. $-5^{\circ}$; sunny; light wind; 4 species, 487 individuals. Rock Dove, 10; Black - billed Magpie, 7; House Sparrow, 20; Snow Bunting, 450.-J. W. Hamilton and son.

RAYMORE, Dec. 27; 10 miles on foot and 15 miles by car; temp. $-5^{\circ}$; wind light; overcast; 16 species, 249 individuals. Ruffed Grouse, 11; Sharptailed Grouse, 12; Rock Dove, 20; Great Horned Owl, 4; Hairy Woodpecker, 3; Downy Woodpecker, 4; Gray Jay, 1; Black-billed Magpie, 5; Common Crow, 1; Black-capped Chickadee, 7; Brown Creeper, 1; Bohemian Waxwing, 3; House Sparrow, 30; Pine Grosibeak, 17; Common Redpoll, 20; Snow Bunting, 100. (Add: Gray Partridge, 30, Dec. 22; Northern Shrike, 1, Jan. 1; Evening Grosbeak, 1, Dec. 25; White-winged Crossbill, 1, Dec. 29).Wayne Harris.

REGINA, Dec. 26; 197 miles by car in 25 party hours, and 59 miles on foot in 10 party hours; temp. $-10^{\circ}$ to $-4^{\circ}$; wind NW $15 \mathrm{mph}$; fog patches in A.M., clear in P.M. and mist over Waterfowl Park; 6 inches of snow; 36 species, 4775 individuals. White Pelican, 1; Mute Swan, 3; Whistling Swan, 5; Canada Goose, 300; Mallard, 525; Gadwall, 1; Canvasback, 2; Common Goldeneye, 7; Ruffed Grouse, 1; Sharptailed Grouse, 11; Gray Partridge, 35; Coot, 1; Rock Dove, 144; Great Horned Owl, 3; Snowy Owl, 8; Saw-whet Owl, 1; Yellow-shafted Flicker, 6; Hairy Woodpecker, 4; Downy Woodpecker, 6; Horned Lark, 13; Black-billed Magpie, 51; Common Crow, 1; Blackcapped Chickadee, 24 ; Brown Creeper, 1; Mockingbird, 1; Robin, 3; Bohemian Waxwing, 1107; Cedar Waxwing, 1; Northern Shrike, 2; Starling, 16; House Sparrow, 2413; Red-winged Blackbird, 1; Common Grackle, 3; Pine Grosbeak, 24; Common Redpoll, 44; Snow Bunting, 6. (Add: Pintail, 1, Dec. 23; Rusty Blackbird, 1, Dec. 21; Evening Grosbeak, 1, Dec. 24).-Marc
Abrioux, Gary Anweiler, Ron Austin, Mrs. J. Bailey, Margaret Belcher, Al Binnie, Carole Binnie, Greg Bobbitt, Frank Brazier, Bill Brownlee, Eric Cooke, Doug Gilroy, Jay Jordan, Jim Jowsey, Harriet Jow sey, George Ledingham, Christine MacDonald, Helen Morrison, Sally Moss, Eric Olesen, Maureen Rever, Joe Roberts, Hugh Smith, Elisabeth Wagner, Holly Wallace. (Al and Betty Binnie, compilers).

SALTCOATS, Dec. $30 ; 3$ miles on foot and 12 by car-from dawn to dusk; temp. $-25^{\circ}$; wind NW $10 \mathrm{mph}$; m'ostly clear; 9 species, 181 individuals. Ruffed Grouse, 1; Sharp-tailed Grouse, 10; Gray Partridge, 6; Downy Woodpecker, 2; Black-billed Magpie, 5; Common Raven, 2; Black-capped Chickadee, 5; House Sparrow, 22; Snow Bunting, 128.-Harold Jowsey, J. Norman Jowsey, J. R. Jowsey (compiler), Eric Upshall.

SASKATOON, Dec. 26 ; 19 party hours and 27 party miles on foot, 28 party hours and 239 party miles by car; temp. $-19^{\circ}$ to $-8^{\circ}$; wind NW 7 to 14 mph; clear; 6 inches of snow; 23 species, 7863 individuals. Mallard, 22; Shoveler, 1; Ruffed Grouse, 4; Sharptailed Grouse, 58; Ring-necked Pheasant, 11; Gray Partridge, 269; Rock Dove, 630; Great Horned Owl, 2; Snowy Owl, 3; Hairy Woodpecker, 2; Downy Woodpecker, 4; Horned Lark, 100; Blue Jay, 21; Black-billed Magpie, 171; Black-capped Chickadee, 41; Bohemian Waxwing, 4299; Cedar Waxwing, 15; Northern Shrike, 2; Starling, 4; House Sparrow, 2010; Pine Grosibeak, 141; Common Redpoll, 22; Snow Bunting, 31. (Add: Common Goldeneye, 1, Dec. 25; Yellow-shafted Flicker, 1, Dec. 24; Brown Creeper, 1, Dec. 23; Evening Grosbeak, Dec. 30). Harold Belcher, Dr. Bruce Brandell, W. J. Brucks, John Cartwright, C. Chamberlin, Pern Cordery, Marie Gillespie, Dr. and Mrs. J. B. Gollop, Vi Harper, Mr. and Mrs. J. D. Hogg, David Houston, Dr. C. J. Houston, Dr. Stuart Houston (compiler), Anna Miller, Arnold Nijssen, Joe Schmidt, John and Stan Shadick, Alan Smith, Fred Waite, J. A. Wedgwood. 
SKULL CREEK, Dec. 29; 42 party miles on foot, 6 by car and 15 by tractor in $2 \frac{1}{2}$ hours; temp. $10^{\circ}$ to $0^{\circ}$; light wind; 20 species, 957 individuals. Golden Eagle, 3; Sharp-tailed Grouse, 124; Ring-necked Pheasant, 9; Gray Partridge, 52; Great Horned Owl, 5; Snowy Owl, 1; Saw-whet Owl, 1; Yellow-shafted Flicker, 1; Hairy Woodpecker, 8; Downy Woodpecker, 7; Horned Lark, 22; Blue Jay, 1; Black-billed Magpie, 130; Black-capped Chickadee, 34; Bohemian Waxwing, 310 ; Northern Shrike, 1; House Sparrow, 96; Pine Grosbeak, 58; Common Redpoll, 20; Snow Bunting, 74. (Add: Bald Eagle, 2, Dec.24).-Wtan Birchall, Henry, Edith and Doug Borman, Austin Drever, Dorothy Eccleston, Phyllis Flagg and Randy and Leslie Flagg, Robert, Patti, Jimmie, and Dougie Mann, Steve Mann (compiler), Peter Swain.

SPIRIT LAKE, Dec. $24 ; 5$ party hours and 5 party miles, and about yards; temp. $9^{\circ}$; clear, windy with drifting snow; 8 inches of snow; 13 species, 113 individuals. Ruffed Grouse, 2; Pileated Woodpecker, 1; Hairy Woodpecker, 8; Downy Woodpecker, 8; Gray Jay, 2; Black-billed Magpie, 3; Black-capped Chickadee, 45; White-breasted Nuthatch, 2; Northern Shrike, 1; House Sparrow, 25; Evening Grosbeak, 2; Pine Grosbeak, 2; Snow Bunting, 12. (Add: Sharp-tailed Grouse, 1, Jan. 1; Great Horned Owl, 1, Dec. 20 and 27). -Iris Anaka, Bill Anaka, Mrs. J. Gunn, Joyce Gunn (compiler).

SWIFT CURRENT, Dec. 29; 5 hours; temp. $20^{\circ}$; wind light; 8 species, 3111 individuals. Sharp-tailed Grouse, 2; Ring-necked Pheasant, 1; Gray Partridge, 27; Horned Lark, 18; Blackbilled Magpie, 10; Bohemian Waxwing, 36; Starling, 17; House Sparrow, 3,000.-Mrs. B. Hare, Mrs. J. MeDaid.

VAL MARIE, Dec. 25; 60 miles by car, 4 miles on foot; temp. $20^{\circ}$ to $0^{\circ}$; cloudy, clearing later; 2 inches of snow; 12 species, 1916 individuals. Golden Eagle, 3; Sharp-tailed Grouse, 2 ; Sage Grouse, 1; Ring-necked Pheas- ant, 12; Long-eared Owl, 1; Flicker, 1; Horned Lark, 170; Black-billed Magpie, 21; Black-capped Chickadee, 3; House Sparrow, 1; Pine Grosbeak, 1; Snow Bunting, 1700. (Add: Gray Partridge, 9; Bohemian Waxwing, 1; Northern Shrike, 1; Starling, 2; Tree Sparrow, 10; Fox Sparrow, 1, all on Dec. 22).—J. David Chandler, Ruth Chandler.

WOLSELEY, Jan. 1; 20 miles by car and around farmyard; temp. $-22^{\circ}$; wind light; 10 species, 319 individuals. Ruffed Grouse, 1; Gray Partridge, 25; Rock Dove, 24; Great Horned Owl, 1; Hairy Woodpecker, 2; Horned Lark, 10; Black-billed Magpie, 3; Blackcapped Chickadee, 3; House Sparrow, 50; Snow Bunting, 200. (Add: Snowy Owl, 1, Dec. 23; Northern Shrike, 1). —J. Donald Hayward.

YELLLOW CREEK, Dec. 31; 4 miles on foot for 3 hours; temp. $-15^{\circ}$; wind E $15 \mathrm{mph}$; overcast with light snow; 17 inches of snow; 7 species, 88 individuals. Hairy Woodpecker, 1; Blackbilled Magpie, 6; Common Raven, 1; Black-capped Chickadee, 13; House Sparrow, 64; Evening Grosbeak, 2; Snow Bunting, 1. (Add: Ruffed Grouse, 4, Jan. 1; Great Horned Owl, 1, Dec. 20; Downy Woodpecker, 1, Dec. 25).Bohdan Pylypec.

YORKTON, Dec. $26 ; 155$ party miles by car and $18 \frac{1}{2}$ party miles on foot; iemp. $-15^{\circ}$ to $-3^{\circ}$; wind WNW 15 mph. in P.M.; 10 inches of snow; 16 species, 305 individuals. Ruffed Grouse, 1; Sharp-tailed Grouse, 24; Gray Partridge 12; Rock Dove, 2; Great Horned Owl, 1; Snowy Owl, 2; Hairy Woodpecker, 7; Dorwny Woodpecker, 1; Blue Jay, 3; Black-billed Magpie, 23; Blackcapped Chickadee, 12; Bohemian Waxwing, 18; House Sparrow, 106; Pine Grosbeak, 1; Common Redpoll, 12; Snow Bunting, 80.-Bob Pearce, Gordon' Pearce, Irving Pearce, Larry Morgotch, Frank Switzer, Harold Wilkinson (Yorkton Natural History Society-senior branch). Rod and Michael Bantjes, Bruce Eckhart, Kerry Koch, Keith and Denyse Monette (Yorkton Natural History Societyjunior branch). 